\title{
Thermomechanical assessment of the effects of a jaw-beam angle during beam impact on Large Hadron Collider collimators
}

\author{
Marija Cauchi* \\ CERN, Geneva, Switzerland and University of Malta, Msida, Malta
}

R. W. Assmann, ${ }^{\dagger}$ A. Bertarelli, F. Carra ${ }^{\ddagger}$ L. Lari, ${ }^{\S}$ and A. Rossi
CERN, Geneva, Switzerland

P. Mollicone and N. Sammut

University of Malta, Msida, Malta

(Received 29 September 2014; published 23 February 2015)

\begin{abstract}
The correct functioning of a collimation system is crucial to safely and successfully operate high-energy particle accelerators, such as the Large Hadron Collider (LHC). However, the requirements to handle highintensity beams can be demanding, and accident scenarios must be well studied in order to assess if the collimator design is robust against possible error scenarios. One of the catastrophic, though not very probable, accident scenarios identified within the LHC is an asynchronous beam dump. In this case, one (or more) of the 15 precharged kicker circuits fires out of time with the abort gap, spraying beam pulses onto LHC machine elements before the machine protection system can fire the remaining kicker circuits and bring the beam to the dump. If a proton bunch directly hits a collimator during such an event, severe beam-induced damage such as magnet quenches and other equipment damage might result, with consequent downtime for the machine. This study investigates a number of newly defined jaw error cases, which include angular misalignment errors of the collimator jaw. A numerical finite element method approach is presented in order to precisely evaluate the thermomechanical response of tertiary collimators to beam impact. We identify the most critical and interesting cases, and show that a tilt of the jaw can actually mitigate the effect of an asynchronous dump on the collimators. Relevant collimator damage limits are taken into account, with the aim to identify optimal operational conditions for the LHC.
\end{abstract}

DOI: 10.1103/PhysRevSTAB.18.021001

PACS numbers: 29.27.-a, 07.05.Tp, 79.20.Ap

\section{INTRODUCTION}

The Large Hadron Collider (LHC) is a circular particle accelerator installed in an underground tunnel with a circumference of $27 \mathrm{~km}$. Two counterrotating beams of protons travel at close to the speed of light in separate beam pipes that are kept at ultrahigh vacuum. The two beams gain energy with every lap until they reach the required maximum energy. The design beam consists of 2808 bunches with a nominal bunch intensity of $1.15 \times 10^{11}$ protons [1]. Moreover, each of the two LHC beam pipes is designed to handle a stored beam energy of up to $362 \mathrm{MJ}$ $\left(3 \times 10^{14}\right.$ protons at $\left.7 \mathrm{TeV}\right)$, for center of mass collision energies up to $14 \mathrm{TeV}$. This design stored energy is, at least, a

*marija.cauchi@cern.ch

Present address: DESY, Germany.

Also at Politecnico di Torino, Turin, Italy.

${ }^{\S}$ Also at IFIC (CSIC-UV), Valencia, Spain.

Published by the American Physical Society under the terms of the Creative Commons Attribution 3.0 License. Further distribution of this work must maintain attribution to the author(s) and the published article's title, journal citation, and DOI. factor of 100 higher than in other hadron machines with superconducting (SC) magnets, thus making the LHC beams highly destructive [2].

Nominally, the core of each bunch has a cross section that can be described by a two-dimensional Gaussian distribution. As the bunches rotate within the LHC ring, particles at the edges of the spatial distribution tend to escape from the proper trajectory, and form a beam halo. Lost beam halo and particle interactions at the experiments make the beam lifetime finite [3]. Beam losses must be reduced as much as possible, and sensitive equipment, particularly the SC magnets, must be protected. Beaminduced magnet quenches [4] and other equipment damage are to be avoided since they might lead to LHC machine downtime, which in turn compromises the time available for collecting physics data.

The LHC uses a multistage collimation system [5] that is $>99 \%$ efficient in removing the beam halo [6]. The collimation system used for the LHC Run 1 (2010-2013) consists of 108 collimators and absorbers. These are installed in seven out of eight LHC interaction regions (IRs) (Fig. 1), as well as in the transfer lines [2]. There are 100 collimators with dual movable jaws in vertical, 


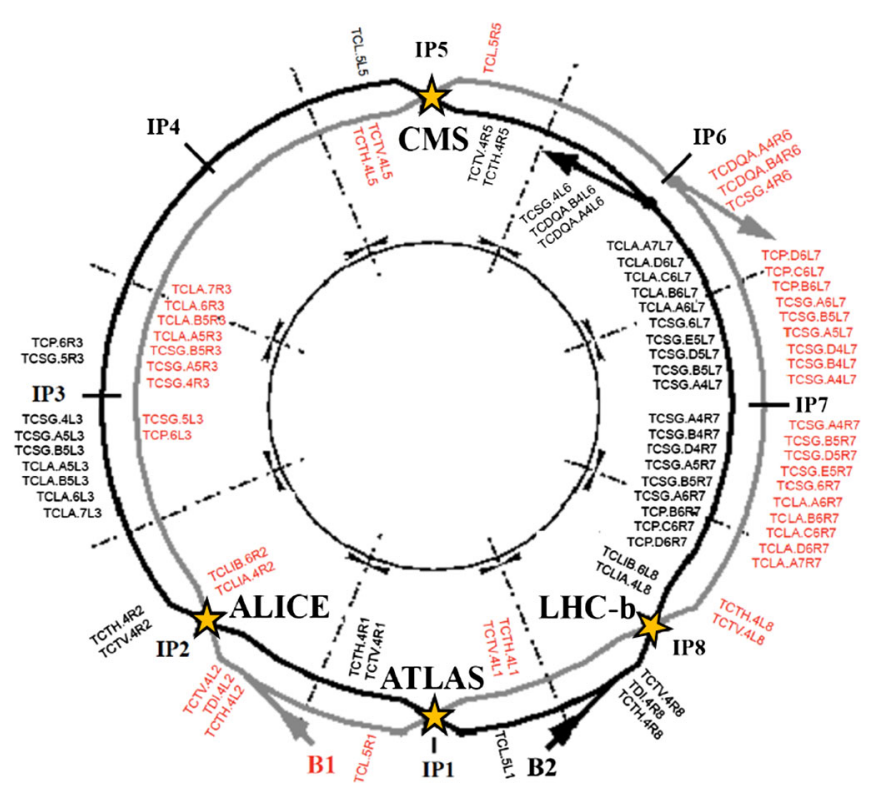

FIG. 1. Sketch of the collimator layout in the LHC IRs during Run 1. Collimators are installed on both beam lines, all around the LHC ring. They are located mainly in IR3 and IR7, where they ensure momentum and betatron cleaning of the particles, respectively. Collimators also protect the four experiments (ATLAS [7], ALICE [8], CMS [9], and LHCb [10]), the beam dump (IR6), and the transfer line regions. Collimators for Beam 1 (red) and Beam 2 (black) are distinguished (adapted from [6]).

horizontal and skew configurations in order to maximize cleaning efficiency all around the particle beam axis. The LHC requires collimation during all stages of operation to protect its elements, unlike previous colliders such as the Tevatron at Fermi National Accelerator Laboratory (Fermilab) in the United States, where the main purpose of collimation was to reduce experimental backgrounds. In the LHC, the collimation system forms an important part of the machine protection system.

Primary collimators (TCPs) have the smallest apertures, followed by secondary collimators (TCSs) and W absorbers (TCLAs) with adjustable gaps (Fig. 2). A three-stage

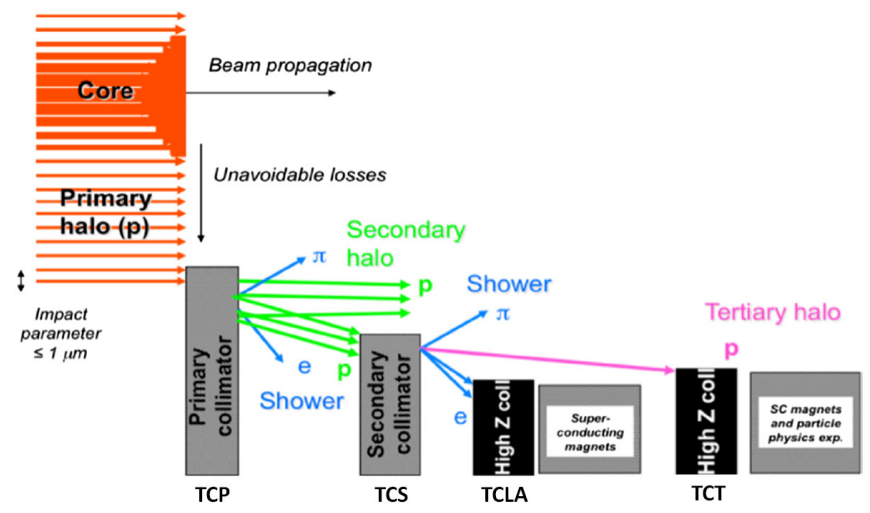

FIG. 2. Qualitative schematic diagram of the LHC multistage collimation system [11]. system of this kind is installed in dedicated warm insertions for $\beta$ (IR7) and off-momentum (IR3) cleaning (Fig. 1).

The TCP and TCS collimators have been designed to withstand beam losses of $90 \mathrm{~kW}$ (corresponding to 1 hour beam lifetime at design conditions), and ten second bursts of losses up to $450 \mathrm{~kW}$. Being closest to the beam, they are at the greatest risk of being hit in an asynchronous beam dump. They are thus made of a carbon fiber composite (CFC) whose high melting temperature and low energy absorption ( $3 \%$ for $1 \mathrm{~m}$ ) make such collimators robust against beam damage. This is, however, achieved at the expense of lower collimation efficiency and higher impedance. The TCLAs are then meant to catch both scattered primary beam and shower debris from upstream collimators. They are made of a W heavy alloy (INERMET® 180) to adequately stop the incoming energy. To protect the TCLAs, the collimator hierarchy must be guaranteed, as the TCLAs would suffer damage if hit by one or several fullenergy full-intensity proton bunches.

In addition to these dedicated collimation insertions, there are also collimators in all the other IRs, except IR4 which houses the SC accelerating radio-frequency cavities and feedback systems. In particular, there are tertiary collimators (TCTs), built with the same design and materials as TCLAs, and installed about $150 \mathrm{~m}$ upstream of the collision points at all the experiments. The TCTs are meant to intercept the tertiary halo close to the particle physics experiments and the sensitive triplet magnets. They provide local protection of the quadrupole triplets in the final focusing system, and are also essential for decreasing the beam-induced backgrounds in the experiments [12].

Other special collimators around the ring intercept debris from beam-beam collisions at the experiments. There are also dump protection collimators (TCDQs) installed at the beam extraction in IR6 to serve as protection against miskicked beams in the case of extraction failures. Similarly, there are injection protection collimators in IR2 and IR8 [1]. Collimators are strategically positioned to provide passive protection for other critical structures such as the SC magnets [13]. If the misinjected or misdumped beams make it past the IR3 and IR7 collimators, they can reach the experiments. In such an event, the TCTs are the last line of defense for the triplet quadrupoles and the particle physics experiments (Fig. 2).

One of the worst accident cases in the LHC corresponds to an asynchronous trigger of the beam dumping system [14], in which one or more high energy density bunches might directly impact a collimator with possible serious consequences [15]. An asynchronous beam dump refers to a situation where one (or more) of the 15 precharged beam kicker circuits fires out of time with the abort gap. Until all the dump kicker magnets are on, the beam may only be partially deflected. While the TCDQ, and the robust TCP and TCS system, are designed to withstand full bunch beam impacts and to protect the cold regions of the LHC and the 
experiments, there may be machine conditions $[16,17]$ that expose the TCTs and thus put them at risk of damage.

This paper evaluates the effectiveness of operating with tilted collimator jaws in case of a direct impact of one full intensity bunch on TCTs, as a consequence of an asynchronous beam dump accident. The TCT design is described, and its thermal and mechanical response, when impacted by 3.5 and $7 \mathrm{TeV}$ bunches of $1.3 \times 10^{11}$ protons, is analyzed through a finite element method (FEM) approach. In particular, we investigate the behavior of the TCTs in case their planar collimating surface is either exactly parallel to the beam or is tilted by -1 or $\pm 5 \mathrm{mrad}$. In all cases, the beam impact parameter to the point of first contact with the collimator is set to $0.5 \mathrm{~mm}$.

\section{BEAM IMPACT ON COLLIMATORS}

\section{A. Tertiary collimator design}

During the LHC Run 1, 38 collimators had $W$-alloy jaws. These are the TCLAs and TCTs described in Sec. I above. This study focuses on TCTs as they are the non-CFC collimation system elements that are mostly at risk of damage in case of an asynchronous beam abort.

A TCT collimator consists of two parallel jaws contained in a vacuum tank, with the beam passing through the center of the jaw gap (Fig. 3). For optimal performance, the jaws have to be centered around the actual beam orbit. Each TCT jaw has a total length of $1.2 \mathrm{~m}(1 \mathrm{~m}$ active length $+0.1 \mathrm{~m}$ tapering at the upstream and downstream parts of the jaw) and consists of five inserts made of INERMET ${ }^{\circledR} 180$. These insert blocks are screwed to a copper housing, which is cooled by $27^{\circ} \mathrm{C}$ water flowing at $25 \mathrm{l} /$ min through an array of square-shaped $\mathrm{Cu}-\mathrm{Ni}$ tubes brazed to the back side of the housing (Fig. 4). Two stepping motors per jaw allow independent adjustment of jaw tilt and jaw position relative to the beam center [18].

\section{B. Thermally induced mechanical effects}

Being in proximity to the beam, the collimator jaws are continuously exposed to direct interaction with high-energy particles. In normal operation, the time constant, which describes variations in thermal load due to particle loss on

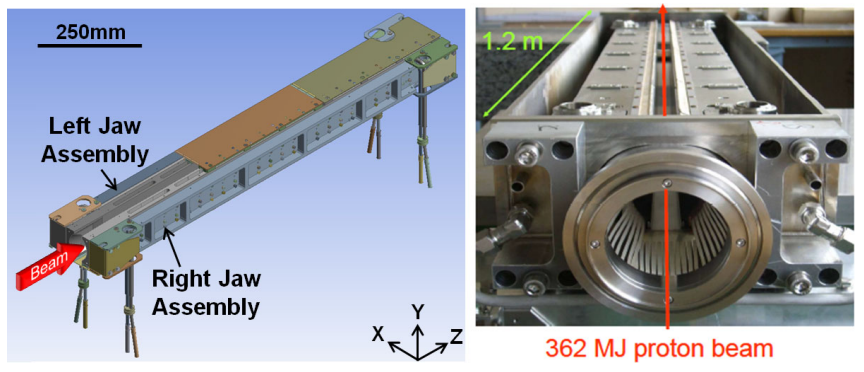

FIG. 3. A horizontal LHC TCT: 3D model (left), and view into the open vacuum tank of the collimator during production (right).

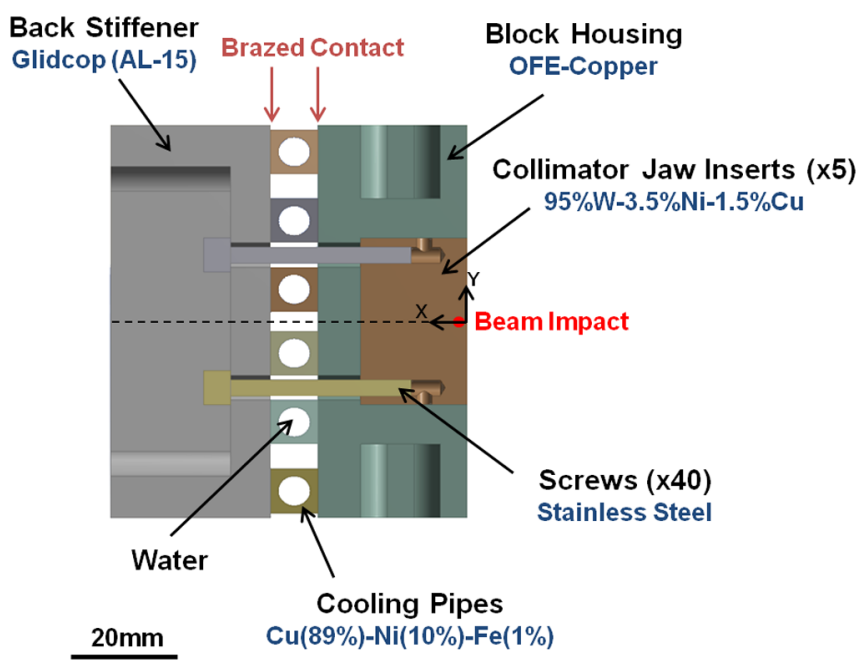

FIG. 4. A detailed cross-sectional view in the $x-y$ plane of the horizontal TCT left jaw assembly, together with the location of the considered beam impact.

the collimator jaws, ranges from seconds to hours. On the other hand, in an asynchronous beam abort accident, the relevant time scale for energy deposition in the jaw material is on the order of $\mu$ s or ns. This very fast energy deposition provokes a thermodynamic response of the hit material, including the development of shock waves within the collimator structure. As the material of the collimator jaw cannot respond to the rapid increase in temperature [19] caused by the hadronic shower, structural deformations can occur [20]. This study investigates collimator damage under such conditions.

\section{STUDIED ACCIDENT CASES}

The probability that an asynchronous beam dump event occurs was originally estimated to be once per year [14]. However, the probability that a TCT is hit directly by a full intensity bunch is lower, as other error conditions [21] must be simultaneously present for this to happen. When an asynchronous abort is detected, the remaining horizontal extraction kickers are fired within $0.9 \mu$ s, and only one bunch should escape the beam dump system. In this context, the present work focuses on the admittedly low probability case of the impact of one bunch on a TCT jaw. If such an accident happens during physics or collimation beam-based alignment setups, it can have serious consequences.

Different jaw error cases have been identified, taking into account conditions when the planar collimating surface of the TCT is either exactly parallel to the beam, or has a slight inclination of a few mrad due to misalignment errors of the collimator installation at the beam line (Figs. 5 and 6). We focus on accidents involving horizontal TCTs due to the fact that a miskick accident can only act on the horizontal plane. 


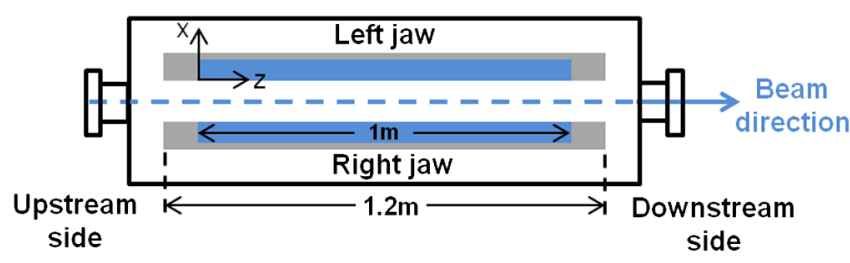

FIG. 5. Top schematic view of a horizontal TCT (not to scale). The blue bars represent the five $W$ jaw inserts (total length $=0.2 \mathrm{~m} \times 5=1 \mathrm{~m}$ ), that are screwed to the copper housing (grey).

All cases studied use a beam bunch of $1.3 \times 10^{11}$ protons with a Gaussian transverse profile of $300 \mu \mathrm{m} \times 300 \mu \mathrm{m}$ root mean square (rms) beam size. A charge of $1.3 \times$ $10^{11}$ protons/bunch [22] constitutes a conservative approach with respect to the nominal bunch intensity. This choice also serves as a good representation of the LHC operational conditions during Run 1, during which the design bunch intensity was surpassed, and the average bunch intensities in 2011 and 2012 were $1.2 \times 10^{11}$ and $1.4 \times 10^{11}$ protons/bunch respectively.

The impact parameter, which is the transverse depth at which the beam first makes contact with the jaw material, is assumed to be $0.5 \mathrm{~mm}$. Studies with an impact parameter of $2 \mathrm{~mm}$ have been carried out in [22,23] in which the full misdirected bunch impacts the bulk material of the jaw inserts. By using an impact parameter of $0.5 \mathrm{~mm}$ in this study, we investigate the effects of grazing of the misdirected bunch on the TCT's planar collimating surface. In this

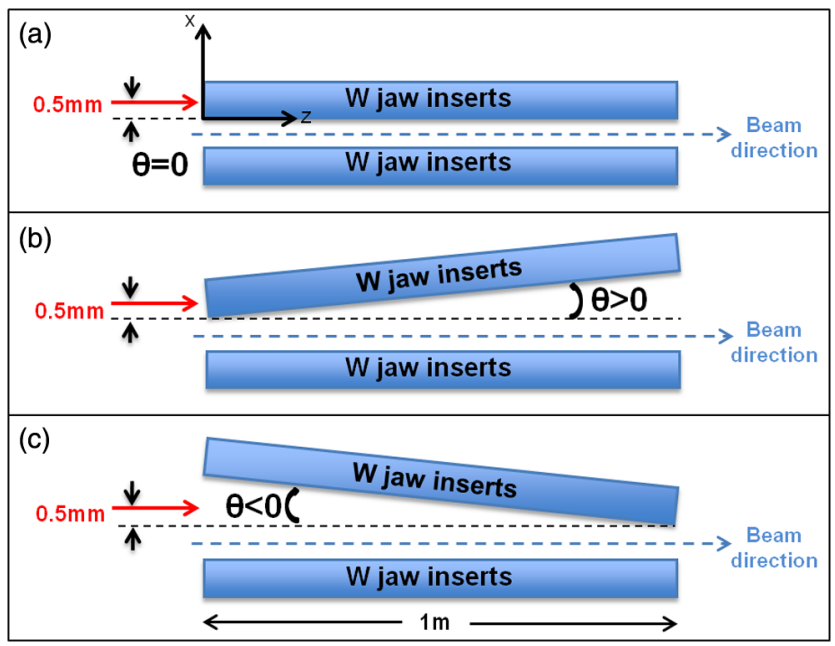

FIG. 6. Schematic diagrams (not to scale) of the studied jaw error cases: an ideal case in which the collimator jaws are perfectly aligned with respect to the beam direction $(\theta=0)(a)$, and cases with a tilt $(\theta>0, \theta<0)$ of one collimator jaw due to misalignment errors (b) and (c). Each blue bar comprises five $\mathrm{W}$ inserts, where the dimensions of each insert are $20 \mathrm{~mm}(x) \times$ $34 \mathrm{~mm}(y) \times 200 \mathrm{~mm}(z)$, while the considered beam impact parameter is $0.5 \mathrm{~mm}$ in the $x$ direction. The sign of the tilt angle is shown, and the dashed black line refers to the collimator position in design orbit.
TABLE I. List of studied jaw error cases. The convention for the positive and negative values of the angle is as depicted in Fig. 6. The LHC operation started at a lower-than-design energy of $3.5 \mathrm{TeV}$ in 2010 , with the goal to reach the design parameters of $7 \mathrm{TeV}$ per beam in the future.

\begin{tabular}{lcccc}
\hline \hline Case & $\begin{array}{c}\text { Energy } \\
{[\mathrm{TeV}]}\end{array}$ & $\begin{array}{c}\text { Angle, } \\
\theta[\mathrm{mrad}]\end{array}$ & $\begin{array}{c}\text { Deposited energy on } \\
1 \text { jaw }[\mathrm{kJ}]\end{array}$ & $\begin{array}{c}\text { TNT equivalent } \\
{[\mathrm{g}]}\end{array}$ \\
\hline 1 & 7 & 0 & 48.50 & 11.56 \\
2 & 7 & +5 & 12.11 & 2.89 \\
3 & 7 & -5 & 10.32 & 2.46 \\
4 & 3.5 & 0 & 23.08 & 5.50 \\
5 & 3.5 & +5 & 6.39 & 1.52 \\
6 & 3.5 & -5 & 5.22 & 1.24 \\
7 & 7 & -1 & 29.73 & 7.09 \\
8 & 3.5 & -1 & 14.18 & 3.38 \\
\hline \hline
\end{tabular}

way, we complement the other studies in analyzing a range of reasonable values for an asynchronous beam dump scenario [5].

A FLUKA jaw-beam angle scanning study is carried out in [21] in order to provide inputs for the ANSYS ${ }^{\circledR}$ [24] calculation. The tilt of one collimator jaw is changed from $\theta=-5 \mathrm{mrad}$ to $\theta=+5 \mathrm{mrad}$ in steps of $1 \mathrm{mrad}$. The tilts of $\pm 5 \mathrm{mrad}$ are studied as limiting cases, and thus this paper presents the cases of $\theta=0 \mathrm{mrad}$ and $\theta= \pm 5 \mathrm{mrad}$ to represent the studied angle range. In addition, a tilt of $-1 \mathrm{mrad}$ is also studied in ANSYS ${ }^{\circledR}$ as the FLUKA study indicates that such a tilt can actually reduce the damage caused by an asynchronous beam dump on the hit collimator.

Table I summarizes the cases studied. A $7 \mathrm{TeV}$ bunch with $1.3 \times 10^{11} \mathrm{p}$ carries $146 \mathrm{~kJ}$ of energy. The lower values in Table I reflect the fact that energy from the shower escapes from the upstream and downstream faces of the collimator jaw. The lower energy deposited for the tilted jaw cases indicates more shower leakage. When the tilt angle is $+5 \mathrm{mrad}$, the beam strikes the upstream face and the nominal exit point is $10 \mathrm{~cm}$ away; when the tilt angle is $R-5 \mathrm{mrad}(-1 \mathrm{mrad})$, the beam strikes the jaw surface $10 \mathrm{~cm}(50 \mathrm{~cm})$ from its downstream end.

\section{NUMERICAL ANALYSIS}

\section{A. Simulation tools}

As the collimator is a multicomponent system composed of different materials and contact interfaces, a numerical FEM approach using the code ANSYS $®$, rather than an analytical analysis, is used to study the response of the collimator to an asynchronous beam abort. This FEM analysis is complemented by nonlinear hydrocode simulations described in [22], that account for phase changes and the presence of shock waves.

The Monte Carlo based statistical code FLUKA [25,26] is used to calculate the thermal load on the $W$ inserts. The full FLUKA shower simulations [21] provide the spatial 
distributions of energy deposition per unit volume per $\mathrm{GeV}$ incident (Fig. 7). A mesh convergence sensitivity study is also carried out in FLUKA, from which it is concluded that a mesh size of $0.1 \mathrm{~mm}(x) \times 0.1 \mathrm{~mm}(y) \times 5 \mathrm{~mm}(z)$ is the minimum required so that a further mesh refinement does not increase the estimated peak power density by $>10 \%$ [27]. The density and phase of $W$ are assumed constant over the duration of the beam impact. A nonlinear, transient analysis (described in Sec. IV B below) is then performed using ANSYS ${ }^{\circledR}$ in order to calculate the jaw temperature and other thermally induced effects as a function of time.
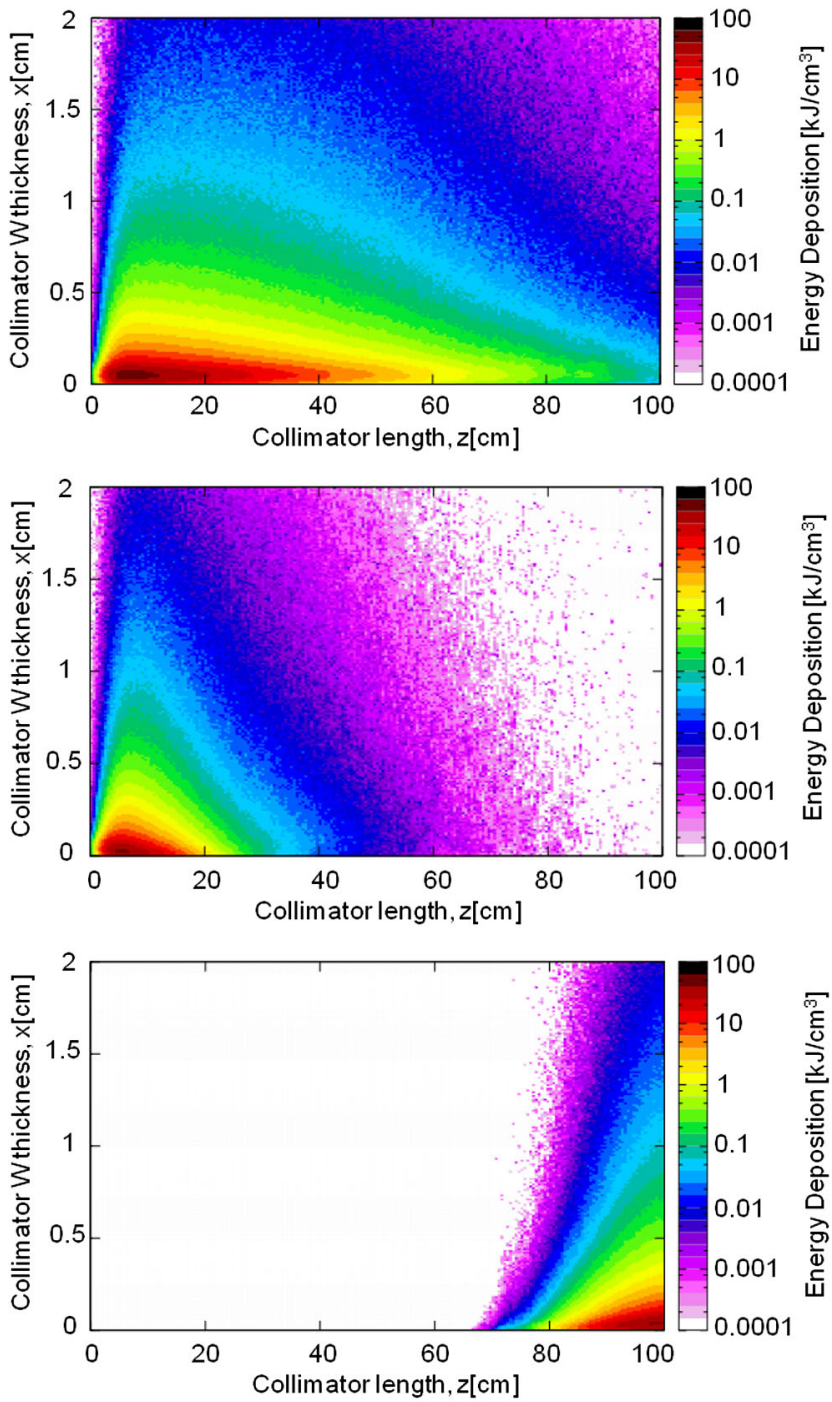

FIG. 7. Comparison of the FLUKA energy deposition maps of the $W$ collimator jaw inserts. All cases consider 1 LHC bunch with $1.3 \times 10^{11}$ protons, a beam size of $0.3 \mathrm{~mm} \times 0.3 \mathrm{~mm}$, and an impact parameter of $0.5 \mathrm{~mm}$. These maps show energy deposition $x-z$ distributions (in $\mathrm{kJ} / \mathrm{cm}^{3}$ ) along the symmetry plane $(Y=0)$, for a $7 \mathrm{TeV}$ particle beam impact case at $\theta=0 \mathrm{mrad}$ (top), $\theta=+5 \mathrm{mrad}$ (middle) and $\theta=-5 \mathrm{mrad}$ (bottom) angles.

\section{B. Finite element modeling}

\section{Geometry and finite element discretization}

Since both the collimator finite element model (Fig. 8) and the FLUKA shower distribution (Fig. 9) are symmetric about the midplane of the $\mathrm{W}$ inserts, only the lower half of the collimator is modeled in order to reduce computational time without compromising accuracy. Furthermore, in the ANSYS ${ }^{\circledR}$ calculation, the contact surfaces are assumed to never slip, meaning that the screwed and brazed surfaces are fixed despite any other deformation of the collimator.

The mesh density of the $W$ jaw for the thermal analysis is set equal to that used in the FLUKA model. This mesh size is implemented within a $5 \mathrm{~mm}(x) \times 5 \mathrm{~mm}(y)$ region around the beam impact in order to correctly capture the

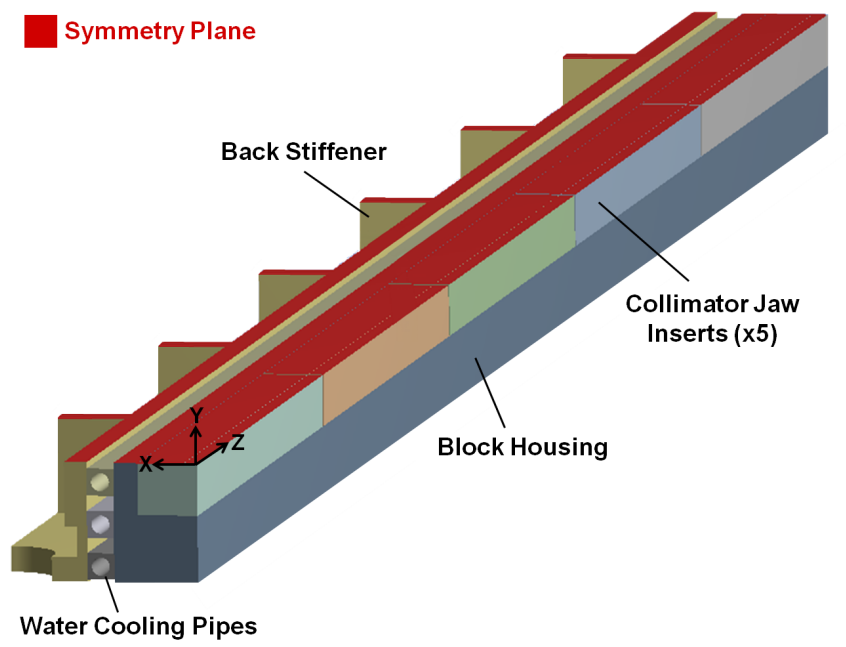

FIG. 8. The lower symmetrical half of a horizontal TCT jaw structure. The symmetry plane ( $x-z$ plane) is shown in red.

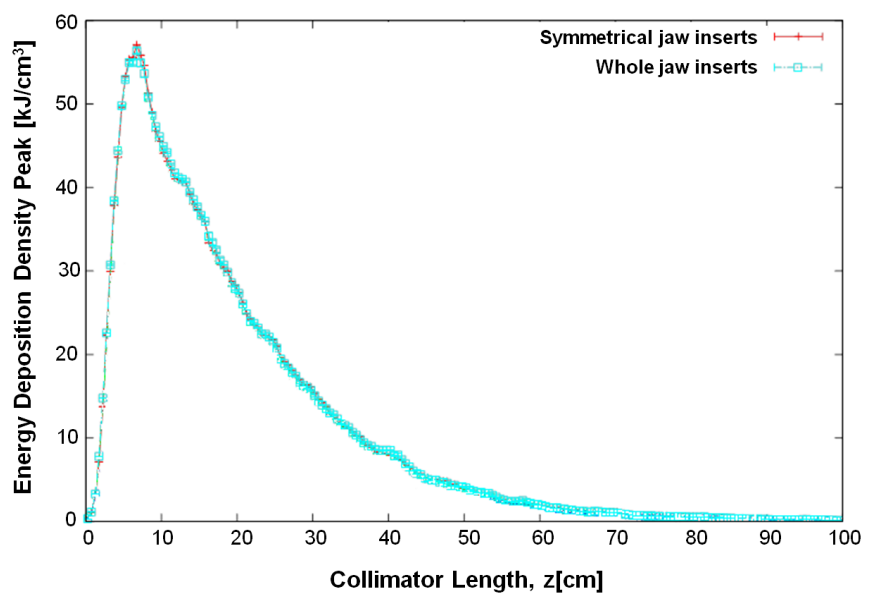

FIG. 9. A comparison of the energy deposition density peak profiles for a whole collimator model and a symmetrical model, with both cases having the same mesh density. It can be concluded that the two models have an identical energy deposition density peak profile, thus justifying the use of symmetry. 

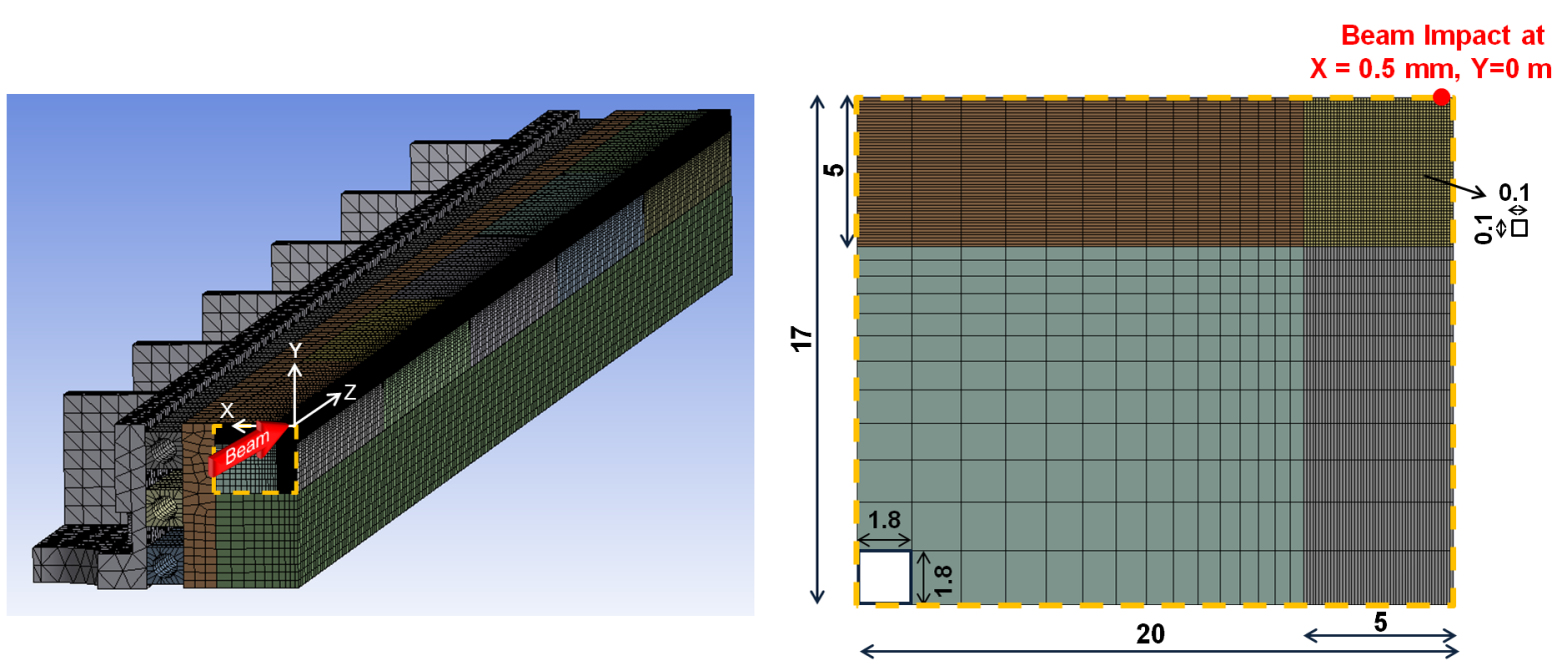

FIG. 10. Finite element discretization of the collimator jaw assembly for the thermal analysis. The discretized collimator model (left), and a detailed cross-sectional $(x-y)$ view of the collimator jaw inserts (right). All dimensions are in mm. The total number of elements used for the thermal analysis model is 1,056,265.

maximum energy deposition on the jaw inserts (Fig. 10). This region of fine mesh is also sufficient for the different jaw error cases. The rest of the jaw inserts, and all the remaining collimator components, are then discretized using a coarser mesh due to computational requirements (Fig. 10). Different meshes are also implemented for the thermal and structural analyses.

\section{Material modeling}

The material of the TCT jaw inserts is a commercial alloy of W, known as INERMET® 180. It is manufactured by liquid-phase sintering of a powder mixture, composed of $W(95 \mathrm{wt} \%), \mathrm{Ni}(3.5 \mathrm{wt} \%)$ and $\mathrm{Cu}(1.5 \mathrm{wt} \%)$ [28]. Temperature-dependent thermal and structural properties are required in FEM calculations with large temperature variations. As these were not available for INERMET ${ }^{\circledR}$ 180 , we decided to use the more defined temperaturedependent material properties of pure $W$ for the jaw inserts (Figs. 11 and 12).

The jaw insert material is thus modeled as an isotropic, homogeneous, elastic-perfectly plastic material with such properties. Given the complexity of the simulations involved, a 5\% error (resulting from using pure $W$ instead of INERMET® 180) is still reasonable, as highlighted in [22]. For property evaluation at element temperatures beyond the supplied tabular range, ANSYS ${ }^{\circledR}$ assumes a constant property at the extreme range value. The other components making up the collimator jaw assembly are also modeled with temperature-dependent thermal and elastic-plastic structural material models.

\section{Loading and boundary conditions}

Accident cases entail rapid energy deposition on the hit structure. In the case of the asynchronous beam dump

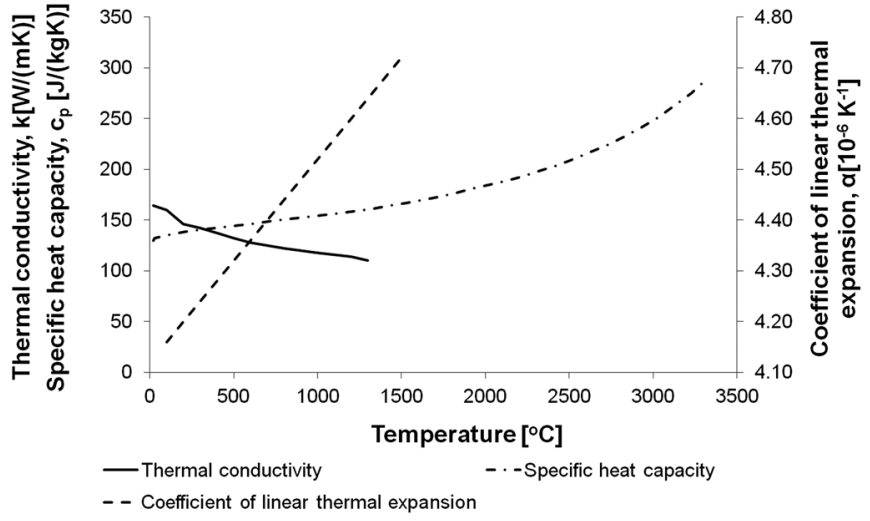

FIG. 11. Temperature-dependent thermal properties of $W$ used for the material model of the collimator jaw inserts [29].

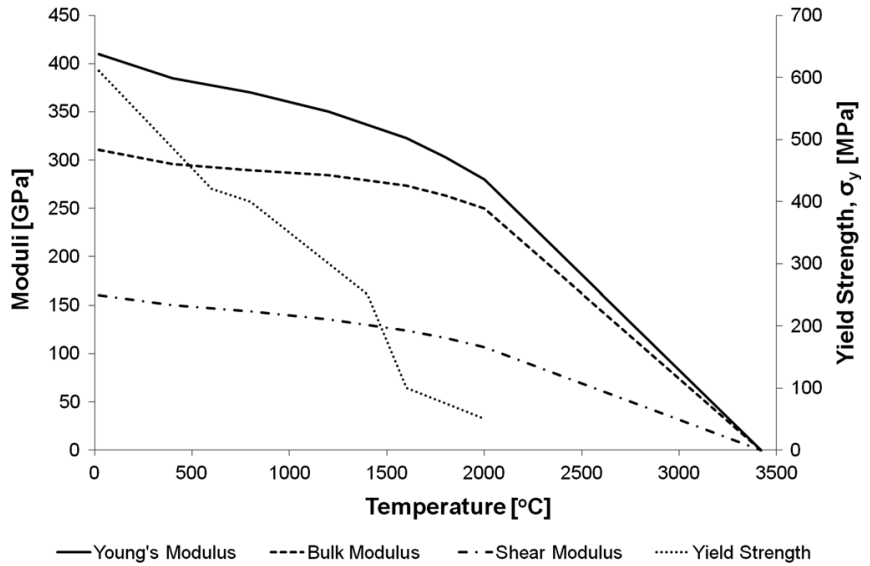

FIG. 12. Temperature-dependent structural properties of $W$ used for the material model of the collimator jaw inserts [29]. 
considered in this study, the thermal shock duration, $\tau_{\text {shock}}$, is equal to the length of one bunch (1 ns). In ANSYS $®$, the thermal load from the beam impact, as calculated by FLUKA, is applied as an instantaneous source of heat within the body of the collimator jaw, and a transient thermal analysis is performed for the whole collimator structure. The heat convection coefficient of the inner wet surface of the cooling pipes is analytically calculated as a function of hydraulic parameters (thermal conductivity, kinematic viscosity) at the water temperature, water flow rate and inner diameter of the pipes, leading to a film coefficient of $13,500 \mathrm{~W} / \mathrm{m}^{2} \mathrm{~K}$ on each pipe. Due to the very short duration of the beam impact, the cooling system does not exchange any heat with the jaw during this time, so that the total energy deposited is equal to that of the beam (Table I).

With regard to structural constraints, the collimator jaw assembly is simply supported at its extremities (Fig. 13), meaning that while the two ends cannot move transversely (toward or away from the beam), one end can elongate (along the beam). The motion in the remaining coordinate is zero by the symmetry condition. The effect of inner pressure due to the water flow on the cooling pipes is also considered. A pressure of 15 bar has been experimentally measured [30], and an equivalent load is applied to the inner surface of the cooling pipes in the finite element model.

As shown by Kalbreier et al. [31], it is possible to assume that no heat diffusion occurs during $\tau_{\text {shock }}$ because the characteristic thermal diffusion time, $\tau_{\text {diffusion, }}$ is much longer than $\tau_{\text {shock }}$. The $\tau_{\text {diffusion }}$ for the jaw inserts (assumed material: pure $W$ ) was found to be $\sim 153 \mu$ s using Eqs. (1) and (2), and considering the transverse edge length of one mesh element in the thermal solution $(0.1 \mathrm{~mm})$ as the typical dimension of the structure. Equations (1) and (2) are given by:

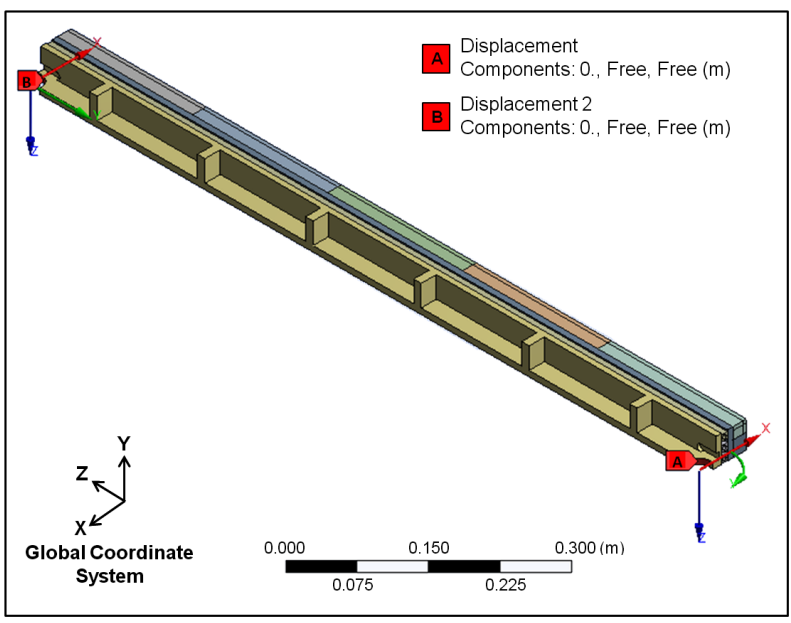

FIG. 13. Back view of the collimator jaw assembly simply supported at its extremities.

$$
\begin{aligned}
\tau_{\text {diffusion }} & =\frac{l^{2}}{\kappa_{c c}}, \\
\kappa_{c c} & =\frac{k}{\rho c_{p}},
\end{aligned}
$$

where $l$ is the typical dimension along which heat diffusion may occur, $\kappa_{c c}$ is the thermal diffusivity, $k$ is the thermal conductivity, $\rho$ is the density and $c_{p}$ is the specific heat capacity at constant pressure.

Given the rapidity of the phenomenon (1 ns), and the typical thermal diffusion times $(\sim 153 \mu$ s for the studied cases), heat conduction plays a minor role on the short time scale. In this case, the thermal deformations are too small to affect the structural response of the structure, resulting in a weakly coupled thermoelastic problem. This means that the nonlinear, transient thermal and structural analyses can be decoupled and sequentially solved with the calculated temperature field used as an input for the evaluation of thermally induced stresses (Fig. 14). This adopted approach compromises the computational time necessary to perform the analysis because very short time steps must be used to maintain the validity of the short time scale assumptions. The choice of the used time steps will be explained later by Eq. (6).

The temperature $\{T\}$ and the displacement $\{u\}$ degrees of freedom (DOFs) must be evaluated by solving both the thermal and structural problems, which are given in matrix form by Eqs. (3) and (4) respectively:

$$
\begin{aligned}
{\left[C_{\text {thermal }}\right]\{\dot{T}\}+\left[k_{\text {thermal }}\right]\{T\}=} & \left\{Q_{\text {heatgen }}\right\}+\left\{Q_{\text {convection }}\right\} \\
& +\left\{Q_{\text {flux }}\right\}, \\
{[M]\{\ddot{u}\}+[C]\{\dot{u}\}+[K]\{u\}=} & \left\{F_{\text {thermal }}\right\},
\end{aligned}
$$

where $\left[C_{\text {thermal }}\right]$ is the thermal specific heat matrix, $\left[k_{\text {thermal }}\right]$ is the thermal conductivity matrix, $\{T\}$ is the temperature DOF vector, $\{\dot{T}\}$ is the time derivative of the temperature

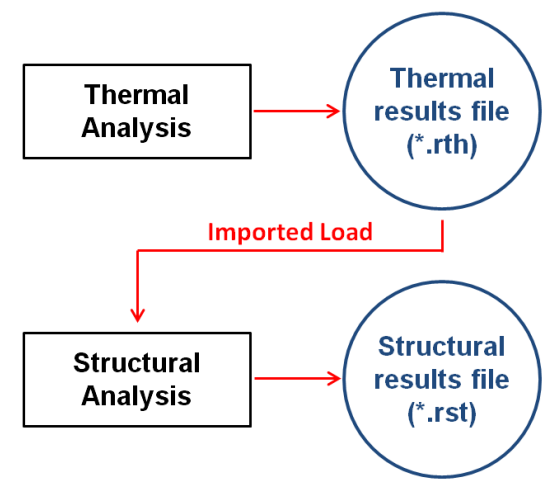

FIG. 14. The data flow for a typical sequentially coupled thermo-structural analysis. Temperatures from the thermal analysis are applied as loads at different time steps in the structural analysis. 
DOF vector, $\left\{Q_{\text {heatgen }}\right\}$ is the heat generation rate load vector, $\left\{Q_{\text {convection }}\right\}$ is the convection load vector, $\left\{Q_{\text {flux }}\right\}$ is the heat flux load vector, $[M]$ is the mass matrix, $[C]$ is the damping matrix, $[K]$ is the structural stiffness matrix, $\{u\}$ is the displacement DOF vector, $\{\dot{u}\}$ and $\{\ddot{u}\}$ are the first and second time derivatives of the displacement DOF vector respectively, and $\left\{F_{\text {thermal }}\right\}$ is the thermal strain load vector.

Integration time steps and mesh size have been carefully chosen in order to catch the correct thermo-structural behavior of the collimator jaw assembly. The rapid temperature increase provokes a dynamic response of the structure in terms of longitudinal and flexural vibrations, as well as propagation of thermal stress waves. The frequency range of these phenomena starts from around $100 \mathrm{~Hz}$, which corresponds to the first period of flexural oscillation of the jaw assembly, as calculated by Eq. (5). Higher modes of longitudinal and transverse vibrations can reach a frequency range on the order of $\mathrm{kHz}$. This wide frequency range has entailed that the duration of the simulation should, at least, be of the order of $100 \mathrm{~ms}$, compared with an integration time step of the order of $1 \mu \mathrm{s}$. Equation (5) is given by:

$$
t_{\text {flex }}=\frac{2}{\pi} \sqrt{\frac{M L^{3}}{E I}}
$$

where $M, L$ and $I$ are the mass, length and cross-sectional inertia of the collimator jaw assembly respectively, and $E$ is an equivalent Young's modulus obtained as an average value between the various materials of the jaw assembly.

Moreover, the integration time step, $\Delta t$, also depends on the mesh size. This is shown by Eq. (6), which represents the Courant criterion [32] for the solution of structural dynamic problems, and is given by:

$$
\Delta t \leq \frac{0.9 L_{\text {mesh }}}{c},
$$

where $L_{\text {mesh }}$ is a typical mesh size in the structural solution, and $c$ is the speed of sound in the jaw insert material $(W)$.

Based on this preliminary analytical estimation, with $L_{\text {mesh }}=1 \times 10^{-3} \mathrm{~m}$ and $c \sim 5180 \mathrm{~m} / \mathrm{s}$ [29], we have fixed the minimum step size to $0.1 \mu \mathrm{s}$ for the structural analysis. Although the implicit method is intrinsically stable, the Courant principle of stability should be applied to avoid numerical damping, which is typical of implicit codes. In ANSYS ${ }^{\circledR}$, Eq. (4) is solved implicitly, which means that $\{u\}$ is determined by computing the inversion matrix of $[K]$, neglecting the mass and damping matrices in the process. The implementation of temperature-dependent material properties in the FEM model introduces a nonlinearity in the system. The matrix $[K]$ becomes a function of the displacement matrix $\{u\}$, and hence methods such as the Newton-Raphson method are used for the solution of nonlinear equations. Meanwhile, each iteration carried out
TABLE II. Load step sequences used for FEM thermal and structural analyses. ITS stands for integration time step. The first load step represents the beam impact. The computational time needed to achieve these structural integration time steps for one simulation is very significant ( $\sim 3$ weeks on a 32 GB RAM 4-core machine with a processor speed of $4.00 \mathrm{GHz}$ ).

\begin{tabular}{lccc}
\hline \hline $\begin{array}{l}\text { Load step } \\
\text { number }\end{array}$ & $\begin{array}{c}\text { Time at end of } \\
\text { load step [s] }\end{array}$ & $\begin{array}{c}\text { Thermal ITS } \\
\Delta t_{\text {thermal }}[\mu \mathrm{s}]\end{array}$ & $\begin{array}{c}\text { Structural ITS } \\
\Delta t_{\text {structural }}[\mu \mathrm{s}]\end{array}$ \\
\hline 1 & $1 \times 10^{-9}$ & $1.2 \times 10^{-4}$ & $1.25 \times 10^{-4}$ \\
2 & $1 \times 10^{-6}$ & 0.1 & 0.1 \\
3 & $1 \times 10^{-4}$ & 2.5 & 1 \\
4 & $2 \times 10^{-3}$ & 20 & 10 \\
5 & 0.02 & 200 & 100 \\
6 & 0.06 & 500 & 250 \\
7 & 0.12 & 500 & 250 \\
\hline \hline
\end{tabular}

by an implicit solver is relatively computationally intensive due to the stiffness matrix inversion operation involved.

The transient thermal and structural load cases are implemented as a sequence of load steps as shown in Table II. The temperature distribution, obtained from the thermal analysis, is applied as a load at different time steps in the structural analysis. ANSYS ${ }^{\circledR}$ linearly interpolates between time step loads, therefore closely following the actual temperature evolution to calculate deformations and other thermally induced effects. Temperature evolution is simulated until $120 \mathrm{~ms}$ according to the typical response time of the structure $\left[t_{\text {flex }}=10 \mathrm{~ms}\right.$ as calculated by Eq. (5)], in order to catch all dynamic phenomena. Due to the unconditional stability of implicit integration schemes, the integration time step can be progressively incremented. In this way, excessive CPU time of calculation is avoided while still ensuring that the higher frequency phenomena are correctly captured on the very short time scale.

The solution is based on three sequential steps: (i) once the heat load is known, the thermal problem can be solved, and the temperature distribution can be calculated as a function of space and time (Sec. VA); (ii) the results of the temperature analysis are used as loads in the structural analysis so that dynamic thermal stresses and displacements can be evaluated (Sec. V B); (iii) a final quasistatic step is necessary in order to calculate potential permanent deformations of the structure once the dynamic response has vanished (Sec. VB). This approach, generally valid for structures submitted to rapid heat loads, has already been successfully applied to the study of the LHC collimators [30].

The LHC collimators should withstand accident scenarios entailing large amounts of very rapidly deposited energy [13]. In this respect, we present a finite element model, based on an implicit algorithm of integration, in order to evaluate both thermally induced vibrations on the short time scale, as well as permanent plastic deformations on the long time scale resulting from the beam impact. The validity of the numerical method used in ANSYS ${ }^{\circledR}$ has 
been confirmed in [23] through a comparison between simulations and experimental measurements on a TCT at the CERN high-radiation to materials facility.

\section{RESULTS AND EVALUATION}

\section{A. Thermal analyses}

A preliminary assessment of the extent of beam-induced damage can be done by evaluating the maximum temperatures reached, and consequently the dimension of the molten region on the jaw inserts. The first step of solution is a transient thermal analysis, where given the energy deposition map, it is possible to calculate the temperature distribution, and its evolution over time. During the $1 \mathrm{~ns}$ beam impact duration, the system receives all the energy, and reaches the maximum temperature on the collimator jaw inserts. In this study, it is assumed that the entire beam is absorbed by the jaw, excluding any hydrodynamic effects. The considered jaw error cases (Table I) give different results, both in terms of peak temperature value as well as its location (Fig. 15). The temperature computation is done starting from the internal energy value, and using the heat capacity and density of the solid material. For this reason, the simulated temperature values are realistic only in the solid material part of the component, and temperatures exceeding the melting temperature are thus shown in dashed lines.

On analyzing the temperature distribution results (Fig. 15), it is clear that in most of the cases, the melting temperature of the jaw insert material is exceeded in certain regions. The molten region caused by the beam impact signifies the formation of a groove on the jaw surface. The high temperatures reached and the extent of the molten

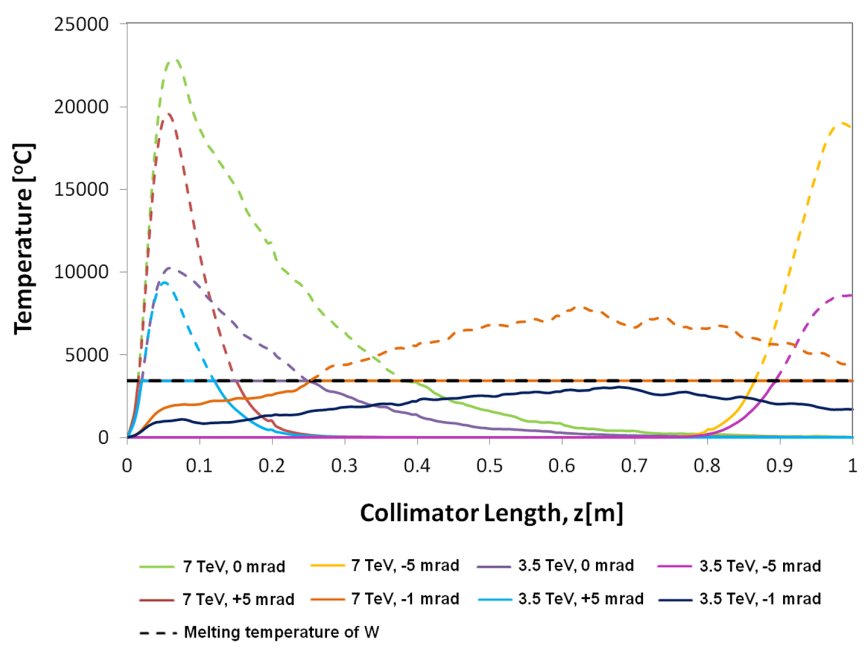

FIG. 15. Temperature peak profiles along the beam direction for the different jaw error cases. The melting temperature of the jaw inserts is marked at $3420^{\circ} \mathrm{C}$, assuming pure $W$ for the jaw insert material. The reason for the dashed graph lines above the melting temperature is that the simulated temperature values are realistic only in the solid material part of the component. region prove the highly destructive nature of the LHC beams.

Once energy deposition distributions are known, the maximum temperature $T_{\max }$ at the end of the heat load deposition can easily be verified (assuming adiabatic conditions) with a simple analytical calculation, given by Eq. (7):

$$
T_{\max }=\frac{P_{\max } \tau_{\text {shock }}}{\rho \int_{T_{\text {ref }}}^{T_{\max }} c_{p}(T) \mathrm{d} T}+T_{\mathrm{ref}}
$$

where $P_{\max }$ is the maximum power density, $\tau_{\text {shock }}$ is the thermal shock duration $(1 \mathrm{~ns})$, and $T_{\text {ref }}$ is the reference temperature $\left(27^{\circ} \mathrm{C}\right)$. Moreover, the density $\left(\rho_{W}=\right.$ $19300 \mathrm{~kg} / \mathrm{m}^{3}$ ) refers to the initial condition of the material (solid state at the nominal density), and the heat capacity at constant pressure $\left(c_{p}\right)$ can be integrated as a function of temperature.

The impact of the beam at different angles also influences the spread in the energy deposition, and thus the cross-sectional temperature distribution on the collimator jaw inserts (Fig. 16). The differences in the extent of the molten region at $7 \mathrm{TeV}$, in particular between the most loaded case (' $7 \mathrm{TeV}, 0 \mathrm{mrad}$ ') and the least loaded case ('7 TeV, -1 mrad'), is evident.

An analysis of the temperature peak profiles also shows that the ' $7 \mathrm{TeV},-1$ mrad' and ' $3.5 \mathrm{TeV},-1$ mrad' cases exhibit different behavior when compared to the other scenarios (Fig. 15). The $0.5 \mathrm{~mm}$ impact parameter and -1 mrad tilt mean that the first point of impact of the

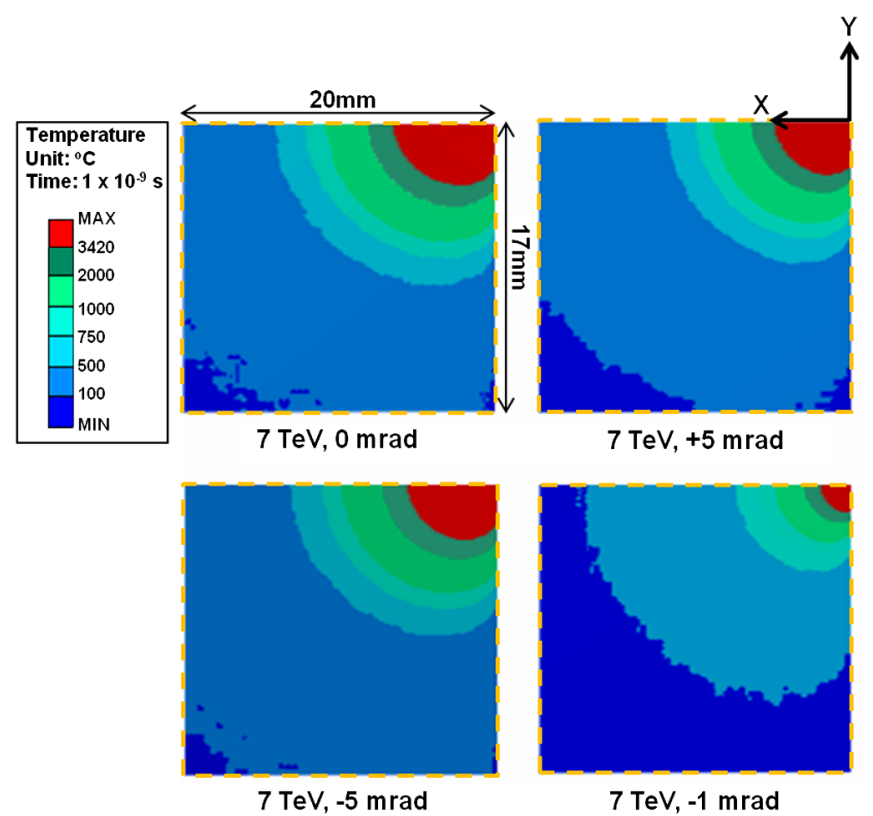

FIG. 16. Comparison of the cross-sectional temperature distribution at the $T_{\max }$-section just after the impact at $7 \mathrm{TeV}$. The red region represents temperatures exceeding the melting temperature of $W\left(3420^{\circ} \mathrm{C}\right)$. The enclosed area is the same as that shown within the dashed line in Fig. 10. 
central $1 \sigma$ of the beam (assumed $0.3 \mathrm{~mm}$ wide rms) is spread between $z=0.2-0.8 \mathrm{~m}$, reducing the peak thermal load. At $\theta=-5 \mathrm{mrad}$, the same first point of impact of the $0.3 \mathrm{~mm} \mathrm{rms}$ beam is spread between $z=0.84-0.96 \mathrm{~m}$. Although the ' $3.5 \mathrm{TeV},-1$ mrad' case involves a larger energy deposition than the ' $3.5 \mathrm{TeV}, \pm 5$ mrad' cases (Table I), the former case has a better temperature distribution along the jaw (Figs. 15, 17 and 18). This was a very interesting observation; hence we decided to focus on the ' $3.5 \mathrm{TeV},-1$ mrad' case for further detailed structural analyses.

In reality, since the material of the jaw inserts is INERMET® 180 and not pure $W$, the copper-nickel matrix melts before the $W$ grains, thus lowering the operating temperature of the whole material. Taking into account this lower melting temperature, the molten region in the '3.5 TeV, -1 mrad' case will still be limited to a small region compared to the other jaw error cases. However, since the jaw insert material is assumed to be pure $W$ for the

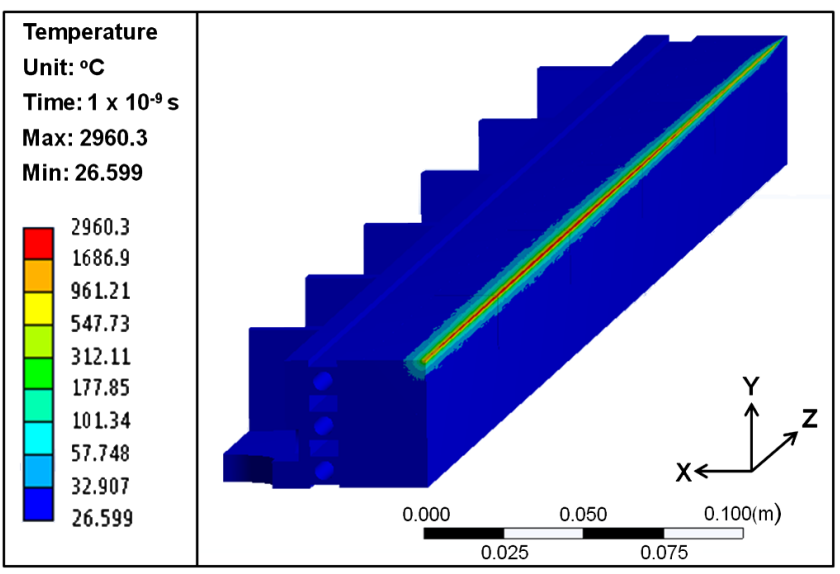

FIG. 17. Temperature distribution for the ' $3.5 \mathrm{TeV},-1 \mathrm{mrad}$ ' case as provoked by the beam impact at $1 \mathrm{~ns}$.

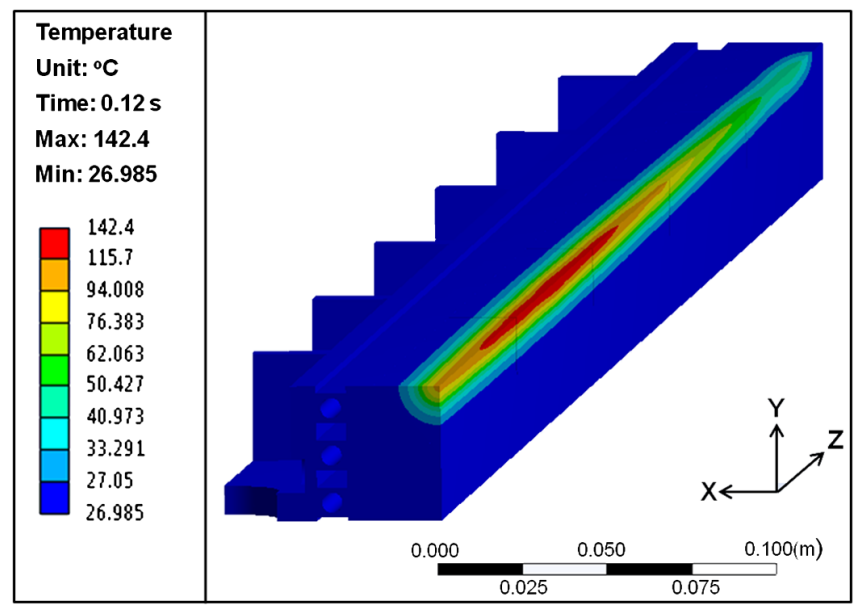

FIG. 18. Temperature distribution for the ' $3.5 \mathrm{TeV},-1 \mathrm{mrad}$ ' case at $120 \mathrm{~ms}$ after the beam impact. purpose of these simulations, the melting temperature $\left(T_{\text {melt }}=3420^{\circ} \mathrm{C}\right.$ for $W$ ) will not be exceeded in any part of the collimator jaw for this particular case. This means that there will be no change of state in the material, making the use of a FEM model with an implicit scheme of integration more appropriate to use.

\section{B. Structural analyses}

In this section, we will present the structural analyses of the collimator structure in the elastic-plastic domain of its constituent materials for the ' $3.5 \mathrm{TeV},-1$ mrad' case. This includes the evaluation of both the dynamic response and the potential permanent damage of the collimator jaw assembly as calculated with ANSYS ${ }^{\circledR}$.

Once the expected temperature increase is known, simple formulas may be applied to estimate the range of compressive stresses as well as to predict plasticization provoked by the thermal shock. Assuming that no expansion occurs, compressive strains, $\epsilon$, and linear elastic stresses, $\sigma^{\text {linear }}$, can be evaluated, using Eqs. (8) and (9) respectively, given by:

$$
\begin{gathered}
\epsilon_{z_{\max }}=-\alpha \Delta T_{\max }, \\
\sigma_{z_{\max }}^{\text {linear }}=-\frac{E \alpha \Delta T_{\max }}{1-2 \nu},
\end{gathered}
$$

where $E$ and $\alpha$ are the Young's modulus and the coefficient of thermal expansion of the jaw insert material respectively, $\Delta T_{\max }=T_{\max }-T_{\text {ref }}$ and $\nu$ is the Poisson's ratio.

The analytical and FEM approaches to find the compressive strains and stresses, which develop within the jaw inserts during the beam impact, are compared (Fig. 19). The compressive strains show good agreement, with both methods giving a maximum compressive longitudinal strain of $\sim 0.012$ at the end of the impact duration.

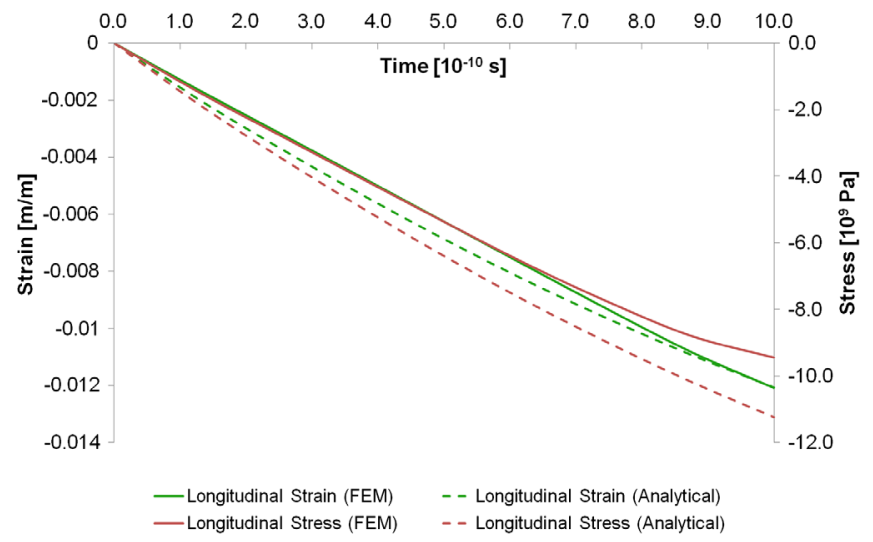

FIG. 19. Maximum compressive longitudinal stresses and strains that develop within the jaw inserts over the duration of the beam impact ( $1 \mathrm{~ns})$. Values obtained as a result of FEM simulations as well as analytically, using Eqs. (8) and (9), are compared. 


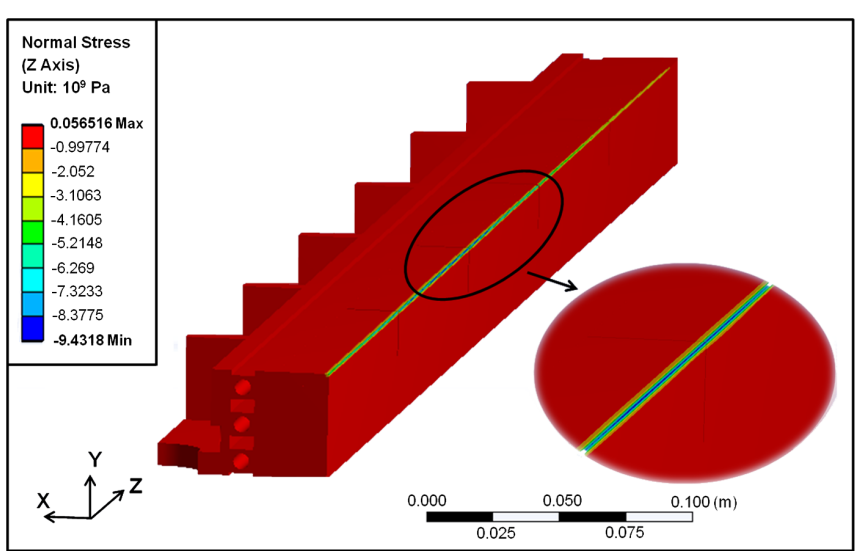

FIG. 20. Longitudinal stresses (z-direction) on the collimator jaw assembly due to the particle beam impact. High compressive stresses dominate the region of the maximum energy deposition. A detailed view is shown in the inset.

Moreover, both approaches give compressive thermal stresses on the order of GPa (Fig. 20). Comparing the temperature distribution (Fig. 17) to the stress distribution (Fig. 20), it can be observed that the region of maximum compressive stresses is the same as the region of maximum temperatures induced during the beam impact. Due to its high temperatures, this region of material tries to expand. However, thermal expansion is prevented by the inertia of the surrounding material, leading to the development of high compressive stresses in the high-temperature region, while tensile stresses develop within the surrounding volume (Fig. 20).

The main frequency of flexural oscillation, as calculated with Eq. (5), is correctly predicted by the numerical simulations. The jaw assembly, hit by the high-energy particle beam, shows a dynamic flexural response with a main frequency of around $95 \mathrm{~Hz}$ (Fig. 21). Furthermore, the jaw assembly vibrates, with an underdamped response,

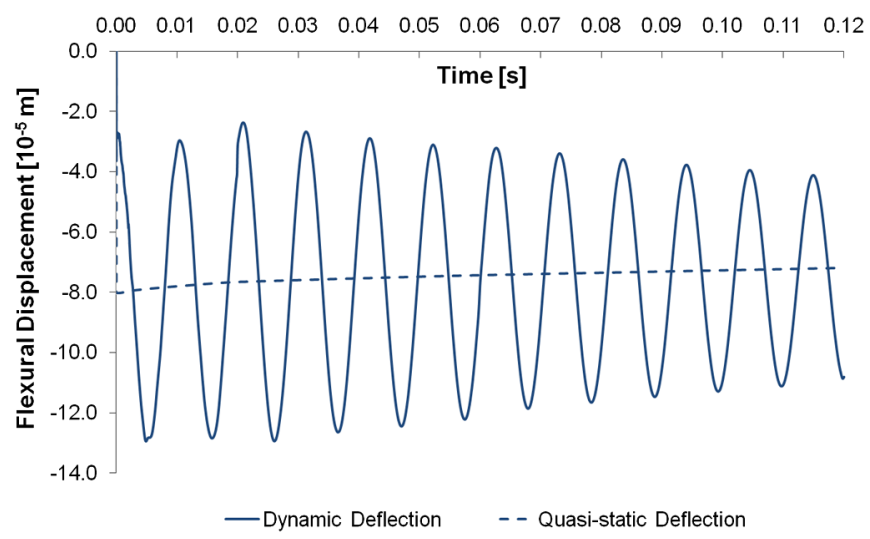

FIG. 21. Dynamic flexural displacement ( $x$-direction) of the collimator jaw assembly at $z=0.42786 \mathrm{~m}$, as provoked by the particle beam impact. The quasistatic deflection due to the thermal bending moment is also shown.

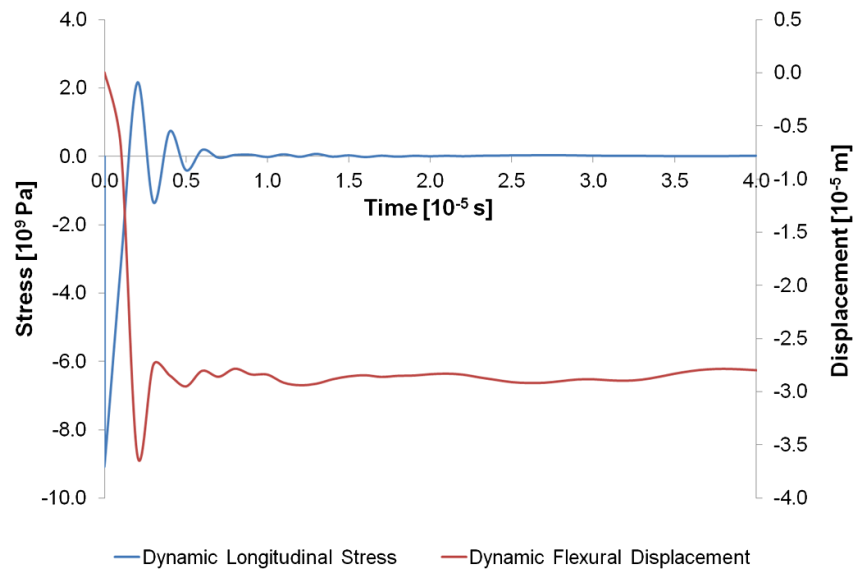

FIG. 22. Dynamic flexural displacement ( $x$-direction) and dynamic longitudinal stress ( $z$-direction) on the collimator jaw assembly at $z=0.42786 \mathrm{~m}$, as provoked by the particle beam impact during the first few $\mu \mathrm{s}$. It can be observed that oscillations start at a frequency range on the order of $\mathrm{kHz}$.

around its quasistatic deflected position. The effect of the temperature distribution (Fig. 17) can be considered as two equivalent dynamic thermal loads: axial force and bending moment. Such an interpretation helps to explain the longitudinal and flexural vibrations, as well as the developed dynamic thermal stresses obtained from the FEM analysis (Figs. 22 and 23).

Thermal stresses on the $W$ jaw exceed the yield strength of the material, meaning that the collimator jaw assembly will remain permanently deformed due to the particle beam impact. Plastic strains are limited to a small region of the collimator jaw inserts (Fig. 24). These residual strains lead to a permanent deformation of $\sim 82 \mu \mathrm{m}$ on the collimator

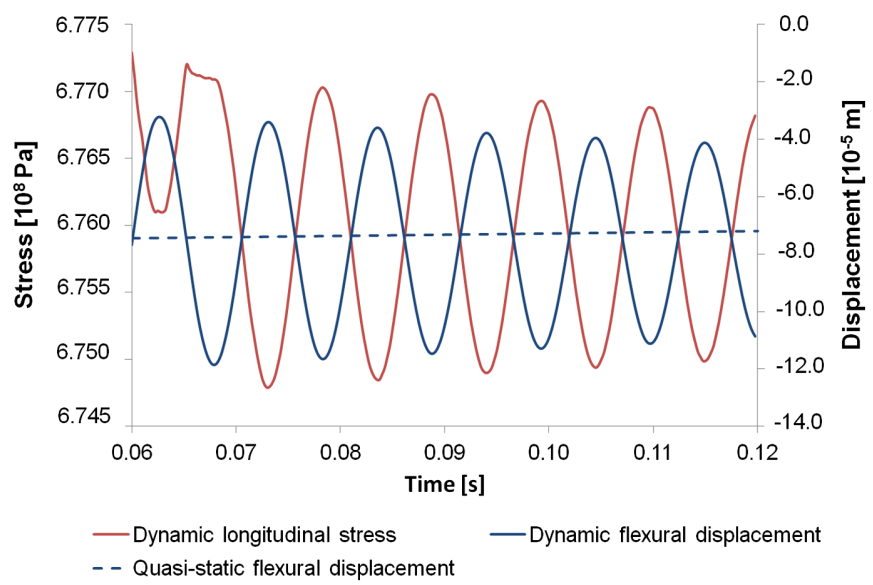

FIG. 23. Dynamic flexural displacement ( $x$-direction) and dynamic longitudinal stress (z-direction) detected on the collimator jaw assembly at $z=0.42786 \mathrm{~m}$ between $t=0.06 \mathrm{~s}$ and $t=0.12 \mathrm{~s}$ after the particle beam impact. It can be observed that oscillations settle at a main frequency of around $95 \mathrm{~Hz}$, which is approximately equivalent to the first period of flexural oscillation of the jaw assembly. 


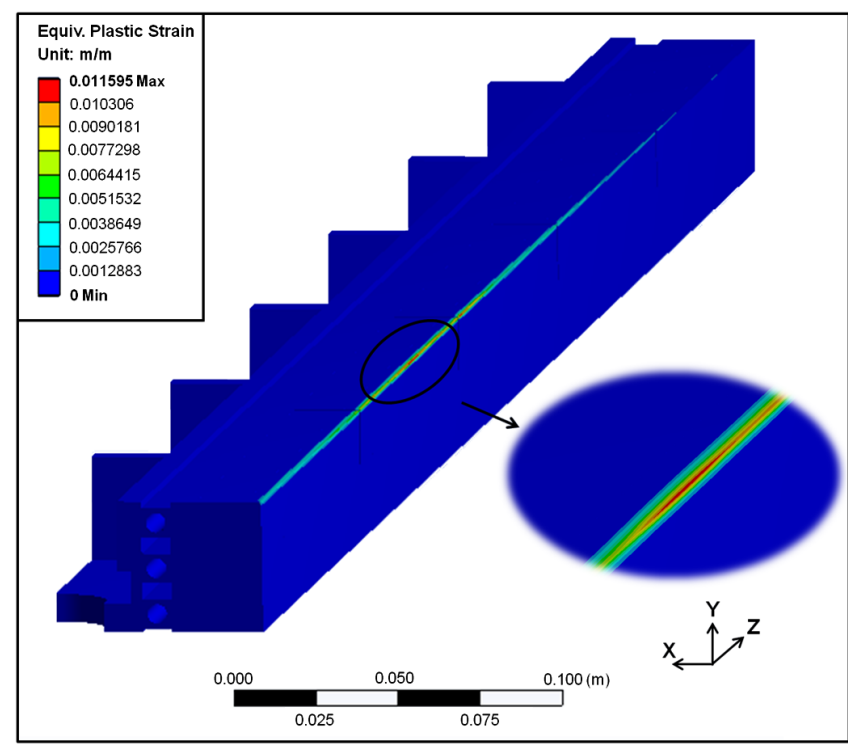

FIG. 24. Equivalent plastic strains on the collimator jaw assembly after the beam impact. A detailed view is shown in the inset.

jaw assembly (Fig. 25). One of the design requirements of LHC collimators is that they must survive proton beam impacts, keeping their correct functionality. The deflection of collimator jaws due to thermal loads induced in nominal steady-state conditions must not exceed the limit of $40 \mu \mathrm{m}$ in order to ensure geometric stability. The accident scenario investigated in this study is of a catastrophic nature when compared to steady-state conditions. In this regard, a jaw

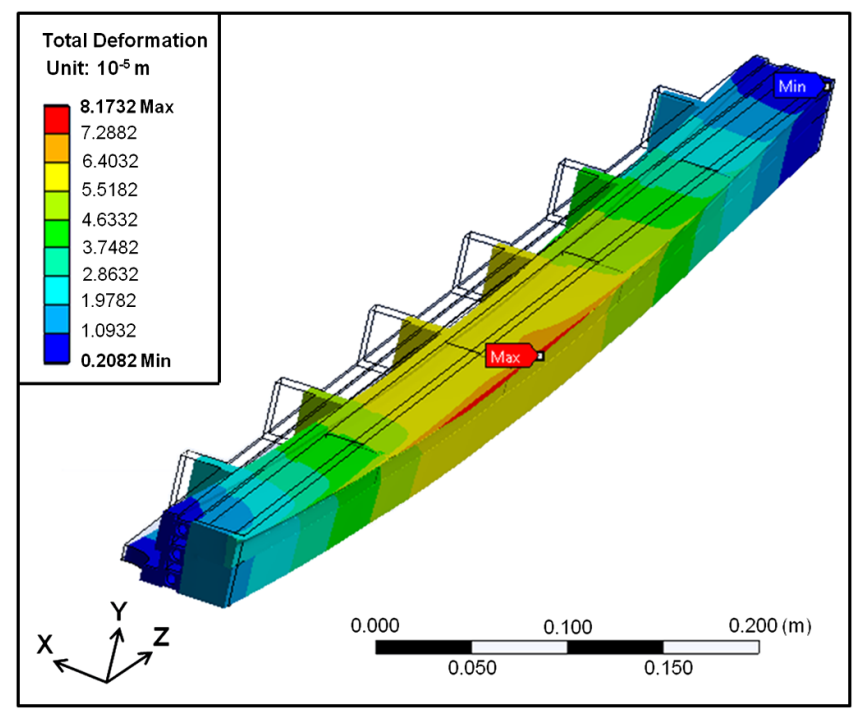

FIG. 25. Total deformation of the collimator jaw assembly resulting from the beam impact. It should be noted that the copper housing, the cooling pipes and the back stiffener must exhibit the same deformation as the jaw inserts as the latter are not allowed to slip in the FEM analysis.

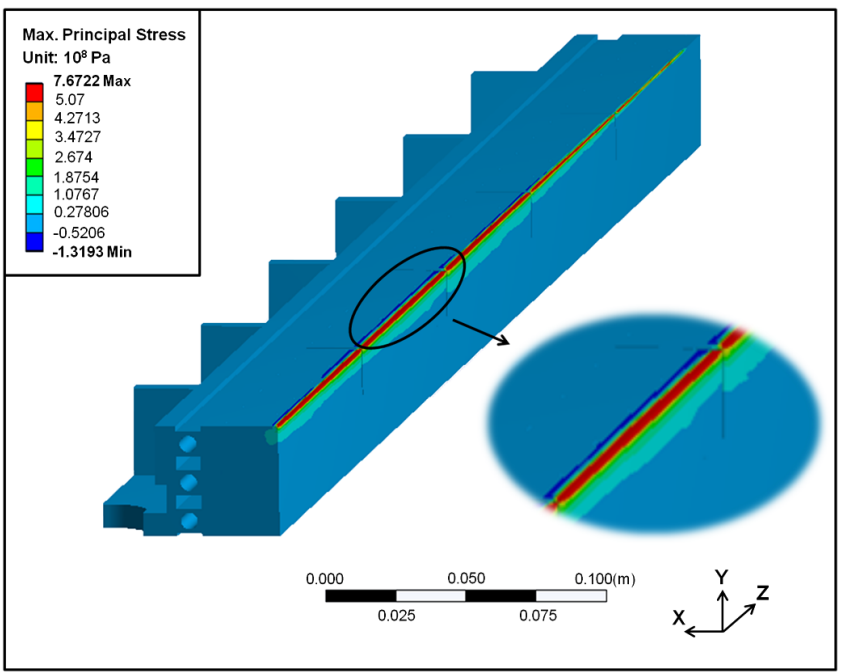

FIG. 26. Contour plot of the maximum principal stress on the collimator jaw assembly. The region of the collimator jaw assembly, where the ultimate tensile strength of $W$ is exceeded, will be subjected to the formation of microcracks and ultimately, spallation. A detailed view is shown in the inset.

deflection of $\sim 82 \mu \mathrm{m}$ resulting from an asynchronous beam dump could still be considered acceptable.

A typical mode of material failure during high velocity impact is spallation. Spallation is the process of internal failure or rupture of condensed media through the nucleation, growth and coalescence of defects, such as microcracks, due to stresses in excess of the tensile strength of the material. The compressive shock wave generated from the impact is reflected as a tensile pulse from the component's free external surface. When this tensile pulse reaches the ultimate tensile strength of the material, a spall is formed. Sequential stages of the damage include the appearance of microcracks, the coalescence of microcracks into one major crack and ultimately, spallation. A contour plot of the maximum principal stress on the collimator jaw indicates that crack formation and damage are limited to a small region (Fig. 26).

\section{CONCLUSIONS}

Predicting the consequences of high-energy particle beams impacting protection devices, such as collimators, is a fundamental issue in the design of state-of-the-art facilities for high-energy particle physics. A challenging simulation campaign, especially in terms of computational resources, has thus been set up. In this study, the thermomechanical response of TCTs in novel jaw error cases involving an asynchronous beam dump, has been evaluated, and we have identified the most interesting load cases for further detailed analyses.

A thermal evaluation of the extent of beam-induced damage has been performed. Comparison of the peak 
temperatures reached, and of the extent of the molten region, indicate that the most loaded case is when the beam impact occurs on jaw inserts that are perfectly aligned with the beam direction $(\theta=0 \mathrm{mrad})$. Significant peak temperatures are also observed when the collimator jaws are slightly inclined. However, we noticed that in the case of a jaw inclination of $-1 \mathrm{mrad}$, no region with very focused energy deposition results from the beam impact, leading to an overall lower peak temperature. Such a tilt, together with an impact parameter of $0.5 \mathrm{~mm}$, mean that the $0.3 \mathrm{~mm} \mathrm{rms}$ beam will strike the jaw surface $50 \mathrm{~cm}$ from its downstream end and that the first point of impact will be spread from $z=0.2$ to $z=0.8 \mathrm{~m}$.

Subsequently, the thermally induced dynamic response of the collimator structure has been studied for the ' $3.5 \mathrm{TeV}$, -1 mrad' case, followed by a quasistatic analysis to calculate potential permanent deformations of the structure once the dynamic response has disappeared. A permanent deformation of $\sim 82 \mu \mathrm{m}$ has been observed on the collimator jaw assembly for the $3.5 \mathrm{TeV},-1 \mathrm{mrad}$ asynchronous beam dump case, and in fact, such damage is much lower than what we would expect of the perfectly aligned ('3.5 TeV, 0 mrad') case. An estimate of the expected damage for the different jaw error cases can be obtained from the peak temperature profiles, as higher peak temperature values will result in larger deformations and thermal stresses.

In conclusion, we have shown that a tilt of the jaw can actually mitigate the effect of the beam-induced damage caused by an asynchronous dump on TCTs. It must however be ensured that the number of escaping highenergy protons in case of a jaw inclination is also studied as such protons can potentially be lost in the SC magnets located downstream of the impacted collimator.

\section{ACKNOWLEDGMENTS}

This research has been supported by CERN (European Organization for Nuclear Research, Geneva, Switzerland), EuCARD 1 and 2, University of Malta and EU Seventh Framework Programme for HiLumi LHC (Grant Agreement No. 284404). The authors would like to highly acknowledge the work of H. Richter and D. Campanini in developing and making available FLUKA-ANSYS interfaces, as well as thank S. Redaelli for various useful inputs and discussions.

[1] O. Brüning, P. Collier, P. Lebrun, S. Myers, R. Ostojic, J. Poole, and P. Proudlock, LHC design report (CERN, Geneva, 2004), http://ab-div.web.cern.ch/ab-div/Publications/ LHC-designreport.html.

[2] R. W. Assmann, O. Aberle, G. Bellodi, A. Bertarelli, C. Bracco, H. Braun, M. Brugger, S. Calatroni, R. Chamizo, A. Dallocchio et al., The final collimation system for the LHC, in Proceedings of the 10th European Particle
Accelerator Conference, Edinburgh, Scotland (EPS-AG, Edinburgh, Scotland, 2006), pp. 986-988.

[3] R. W. Assmann, F. Schmidt, F. Zimmermann, and M. Zorzano-Mier, Equilibrium beam distribution and halo in the LHC, in Proceedings of the 8th European Particle Accelerator Conference, Paris, France (EPS-IGA and CERN, Geneva, 2002), pp. 1326-1328.

[4] J. Jeanneret, D. Leroy, L. Oberli, and T. Trenkler, Quench Levels and Transient Beam Losses in LHC Magnets (CERN, Geneva, 1996), http://cds.cern.ch/record/308241.

[5] O. Brüning, P. Collier, P. Lebrun, S. Myers, R. Ostojic, J. Poole, and P. Proudlock, LHC Design Report-Volume 1, Chapter 18: Beam Cleaning and Collimation System (CERN, Geneva, 2004), http://ab-div.web.cern.ch/ab-div/ Publications/LHC-designreport.html.

[6] C. Bracco, Commissioning scenarios and tests for the LHC collimation system, Ph.D. thesis, Ecole Polytechnique, Lausanne, 2009, http://cds.cern.ch/record/1174254/.

[7] ATLAS Collaboration, The ATLAS experiment at the CERN large hadron collider, JINST 3, S08003 (2008).

[8] ALICE Collaboration, The ALICE Experiment at the CERN LHC, JINST 3, S08002 (2008).

[9] CMS Collaboration, The CMS experiment at the CERN LHC, JINST 3, S08004 (2008).

[10] LHCb Collaboration, The LHCb Detector at the LHC, JINST 3, S08005 (2008).

[11] R. W. Assmann, Collimation for the LHC high intensity beams, in Proceedings of the 46th ICFA Advanced Beam Dynamics Workshop on High-Intensity and High-Brightness Hadron Beams (HB2010), Morschach, Switzerland (PSI, Villigen, 2010), pp. 21-33.

[12] R. Bruce, R. W. Assmann, V. Boccone, G. Bregliozzi, H. Burkhardt, F. Cerutti, A. Ferrari, M. Huhtinen, A. Lechner, Y. Levinsen, A. Mereghetti, N. Mokhov, I. Tropin, and V. Vlachoudis, Sources of machine-induced background in the ATLAS and CMS detectors at the CERN large hadron collider, Nucl. Instrum. Methods Phys. Res., Sect. A 729, 825 (2013).

[13] R. W. Assmann, I. Baishev, M. Brugger, L. Bruno, H. Burkhardt, G. Burtin, B. Dehning, C. Fischer, B. Goddard, E. Gschwendtner et al., Requirements for the LHC collimation system, in Proceedings of the 8th European Particle Accelerator Conference, Paris, France (Ref. [3]), pp. 197-199.

[14] B. Goddard, R. W. Assmann, E. Carlier, J. Uythoven, J. Wenninger, and W. Weterings, Protection of the LHC against Unsynchronised Beam Aborts, in Proceedings of the 10th European Particle Accelerator Conference, Edinburgh, Scotland (Ref. [2]), pp. 1514-1516.

[15] R. W. Assmann, B. Goddard, E. B. Vossenberg, and E. Weisse, The Consequences of Abnormal Beam Dump Actions on the LHC Collimation System (CERN, Geneva, 2002), https://cds.cern.ch/record/691845.

[16] R. Bruce, R. W. Assmann, F. Burkart, M. Cauchi, D. Deboy, L. Lari, S. Redaelli, A. Rossi, B. Salvachua, G. Valentino, and D. Wollmann, Collimation settings and performance in 2011 and 2012, in Proceedings of Chamonix 2012 Workshop on LHC Performance, Chamonix, France (CERN, Geneva, 2012), pp. 183-188. 
[17] L. Lari, R. W. Assmann, V. Boccone, R. Bruce, F. Cerutti, A. Rossi, V. Vlachoudis, A. Mereghetti, and A. Faus-Golfe, Accelerator physics studies on the effects from an asynchronous beam dump onto the LHC experimental region collimators, in Proceedings of 3rd International Particle Accelerator Conference, New Orleans, Louisiana, USA (IEEE, Piscataway, NJ, 2012), Vol. C1205201, pp. 547-549.

[18] A. Bertarelli, O. Aberle, R. W. Assmann, E. Chiaveri, T. Kurtyka, M. Mayer, R. Perret, and P. Sievers, The mechanical design for the LHC collimators, in Proceedings of the 9th European Particle Accelerator Conference, Lucerne, Switzerland (EPS-AG, Lucerne, 2004), pp. 545-547.

[19] N. Tahir, V. Fortov, B. Goddard, D. Hoffmann, V. Kain, I. Lomonosov, A. Piriz, R. Schmidt, A. Shutov, and M. Temporal, Impact of a 7-TeV/c large hadron collider proton beam on a copper target, J. Appl. Phys. 97, 083532 (2005).

[20] P. Sievers, Elastic Stress Waves in Matter due to Rapid Heating by an Intense High-energy Particle Beam (CERN, Geneva, 1974), http://cds.cern.ch/record/416930.

[21] L. Lari, R. W. Assmann, M. Cauchi, A. Rossi, and A. Faus-Golfe, Improved robustness of the LHC collimation system by operating with a jaw-beam angle, in Proceedings of 3rd International Particle Accelerator Conference, New Orleans, Louisiana, USA (Ref. [17]), Vol. C1205201, pp. 553-555.

[22] A. Bertarelli, V. Boccone, F. Carra, F. Cerutti, A. Dallocchio, N. Mariani, L. Peroni, and M. Scapin, Limtis for Beam Induced Damage: Reckless or too Cautious?, in Proceedings of Chamonix 2011 Workshop on LHC Performance, Chamonix, France (CERN, Geneva, 2011), pp. 183-188.

[23] M. Cauchi, O. Aberle, R. W. Assmann, A. Bertarelli, F. Carra, K. Cornelis, A. Dallocchio, D. Deboy, L. Lari, S. Redaelli, A. Rossi, B. Salvachua, P. Mollicone, and N. Sammut, High energy beam impact tests on a LHC tertiary collimator at the CERN high-radiation to materials facility, Phys. Rev. ST Accel. Beams 17, 021004 (2014).

[24] ANSYS® Mechanical, Academic Research, Release 12.1, Help System, Workbench User's Guide, 2009, http:// orange.engr.ucdavis.edu/Documentation 12.1/121/wb2 _help.pdf.

[25] A. Ferrari, P. R. Sala, A. Fassò, and J. Ranft, FLUKA: A Multiparticle Transport Code (Program Version 2005) (CERN, Geneva, 2005), http://www.slac.stanford.edu/ cgiwrap/ getdoc/slac-r-773.pdf.

[26] G. Battistoni, F. Cerutti, A. Fassò, A. Ferrari, S. Muraroi, J. Ranft, S. Roesler, and P. Sala, The FLUKA code: Description and benchmarking, AIP Conf. Proc. 896, 31 (2007).

[27] L. Lari, L. Rivkin, and V. Vlachoudis, Beam-machine interaction studies for the phase II LHC collimation system, Ph.D. thesis, Ecole Polytechnique, Lausanne, 2010, https:// cds.cern.ch/record/1340231/.

[28] Plansee GmbH, DENSIMET® and INERMET® Tungsten Alloys, http://www.plansee.com/en/Materials-Tungsten -heavy-alloys-1577.htm.

[29] Plansee GmbH, Tungsten-material properties and applications, http://www.plansee.com/en/Materials-Tungsten $-403 . h t m$.

[30] A. Dallocchio, Study of thermo-mechanical effects induced in solids by high energy particle beams: Analytical and numerical methods, Ph.D. thesis, Polytechnic University of Turin, Turin, 2008, https://cds.cern.ch/record/1314219/.

[31] W. Kalbreier, W. Middelkoop, and P. Sievers, External targets at the SPS (CERN, Geneva, 1974), https://cds.cern .ch/record/414673/.

[32] R. Courant, K. Friedrichs, and H. Lewy, On the partial difference equations of mathematical physics, IBM J. Res. Dev. 11, 215 (1967). 